\title{
Modulated-temperature thermomechanical analysis
}

\author{
Duncan M. Price* \\ IPTME, Loughborough University, Loughborough LE11 3TU, UK
}

Received 12 September 1998; accepted 19 November 1998

\begin{abstract}
The application of modulated-temperature programming to thermomechanical analysis affords a method for separating the reversible nature of thermal expansion from irreversible deformation arising from creep under the applied load or changes in dimensions due to relaxation of orientation. Measurements may be made under tension (for thin films and fibres) or compression (for self-supporting specimens). The treatment of data is similar to that employed in modulated-temperature DSC (MTDSC). The superposition of a dynamic load in addition to a modulated-temperature program leads to modulatedtemperature dynamic mechanical analysis. (C) 2000 Elsevier Science B.V. All rights reserved.
\end{abstract}

Keywords: Modulated-temperature TMA; Orientation; Creep

\section{Introduction}

The temperature and energy changes associated with thermally activated processes in materials have long been measured by differential scanning calorimetry (DSC). The introduction of modulated-temperature DSC (MTDSC) has allowed the separation of thermally reversible and irreversible (under the conditions of the experiment) events which occur during the course of such measurements [1-4]. A sine wave is the most common heating profile although other waveforms have been used and a variety of data analysis methods have been described [5,6]. Recently, modulated-temperature programs have been used in thermogravimetry to study the thermal degradation behaviour of materials [7].

DSC measures the change in enthalpy of a material as it is subjected to a temperature program. Thermomechanical analysis (TMA), on the other hand, moni-

\footnotetext{
*Tel.: +44-1509-223332; fax: +44-1509-223332. E-mail address: d.m.price@lboro.ac.uk (D.M. Price)
}

tors the change in length (volume) with temperature. By direct analogy to the equations describing MTDSC [1-4], the rate of change of sample length with respect to time $(\mathrm{d} L / \mathrm{d} t)$ measured by thermomechanical analysis (TMA) can be divided into two components:

$\frac{\mathrm{d} L}{\mathrm{~d} t}=\alpha \frac{\mathrm{d} T}{\mathrm{~d} t}+f(t, T)$

where $\alpha$ is the thermal expansion coefficient $(\mathrm{d} L / \mathrm{d} T)$ and $f(t, T)$ encompasses changes in dimension (length or thickness) which occur due to relaxation of stresses in the sample or deformation under the applied load in the case of TMA.

Jaffe [8] has discussed the nature of reversible and non-reversible length changes on heating for oriented polymers. Whilst all materials generally have a positive coefficient of volume expansion, the inherent anisotropy of ordered polymer chains means that the linear thermal expansion coefficient along the chain axis may be negative. Furthermore, the metastable nature of most oriented polymer structures results in relaxation to the disordered state on heating above $T_{\mathrm{g}}$. Hence, any change in specimen length with 
temperature will not be reversible until structural equilibrium is achieved. Trznadel and Krysewski [9] reiterate this concept in a review of thermal shrinkage of oriented polymers. Thermal expansion is reversible (even if the sign of the coefficient of thermal expansion is negative) but any shrinkage is permanent since it results in an increase in entropy due to loss of order in the matrix. Other irreversible dimensional changes can occur: such as creep under the applied load during a measurement. The application of modulated-temperature programming to TMA might afford a means of separating these two effects.

\section{Experimental}

Experiments were carried out using a Shimadzu TMA-50. Measurements were run either in tension (static $1 \mathrm{~g}$ load) or compression (static or dynamic sinusoidal loading $(0.1 \mathrm{~Hz})$ on a $3 \mathrm{~mm}$ diameter flatended probe). A 'modulated' temperature program was synthesised by using a series of heat-isotherm or heat-isotherm-cool-isotherm cycles $[10,11]$. Typical modulation periods ranged from 90 to $300 \mathrm{~s}$ with amplitudes of $1-3^{\circ} \mathrm{C}$ and underlying heating rates between 0.5 and $2^{\circ} \mathrm{C} / \mathrm{min}$.
Deconvolution of the TMA signals (time, temperature and length) was carried out according to the method disclosed by Reading [12]. The algorithm applied a linear least-squares fit to the rate of length change versus heating rate over one period in accordance with the equation above. An adjustable parameter, the phase lag, is introduced to accommodate the delay between the heating rate and thermal response of the system. This is determined by shifting the rate of length change data one point at a time with respect to the heating rate until the best fit is achieved. This 'offline' deconvolution approach is robust, but computationally expensive - typical data files took several hours to analyse.

\section{Results and discussion}

Fig. 1 shows the raw data (temperature and length) and their first derivatives (with respect to time) for a sample of poly(ethylene terephthalate) (PET) film (ICI Melinex ${ }^{\mathbb{R}}$ ). The deconvolution procedure gives the average rate of length change $(\mathrm{d} L / \mathrm{d} t)$, the reversing rate of length change (rev. $\mathrm{d} L / \mathrm{d} T$ ) and the phase lag between the heating program and sample response (Fig. 2). The phase lag can be employed to separate the reversing rate of length change into its in-phase and

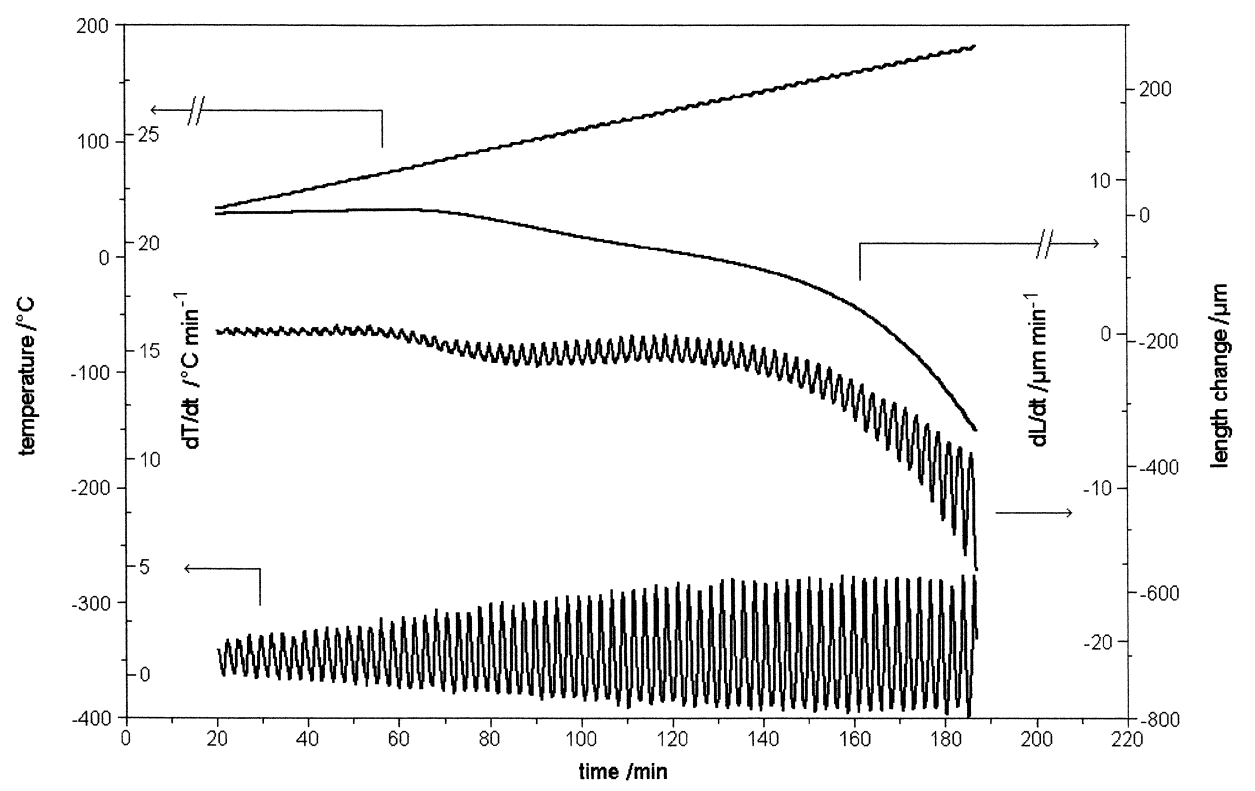

Fig. 1. Raw data from MTTMA experiment on PET film (top — bottom: temperature, length change, $\mathrm{d} L / \mathrm{d} t, \mathrm{~d} T / \mathrm{d} t$ ). 


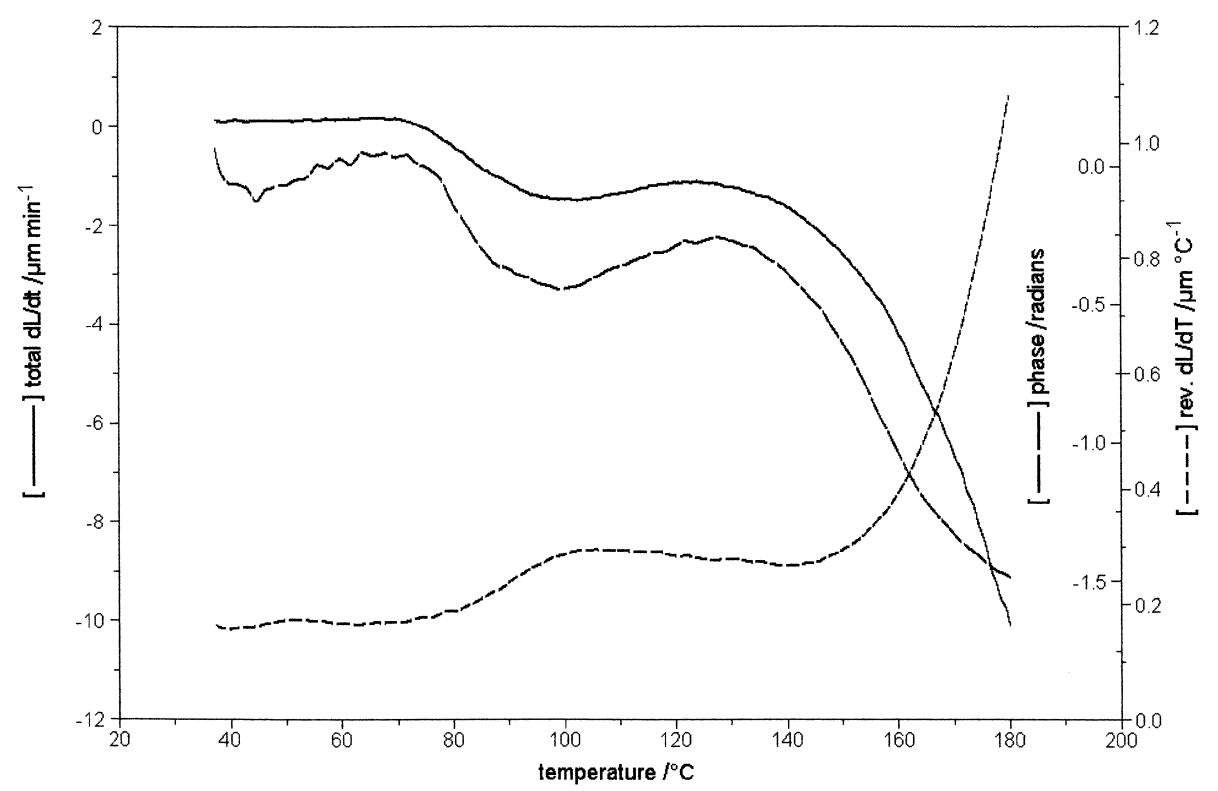

Fig. 2. Deconvoluted data from Fig. 1.

out-of-phase components (Fig. 3) in the same way as can be done for heat capacity measured by MTDSC $[13,14]$. The in-phase response exhibits the usual increase in thermal expansion coefficient through the glass transition, whereas the out-of-phase response contains the kinetic component of the shrinkage due to relaxation of orientation. Conventional TMA is unable to monitor the thermal expansivity of oriented PET

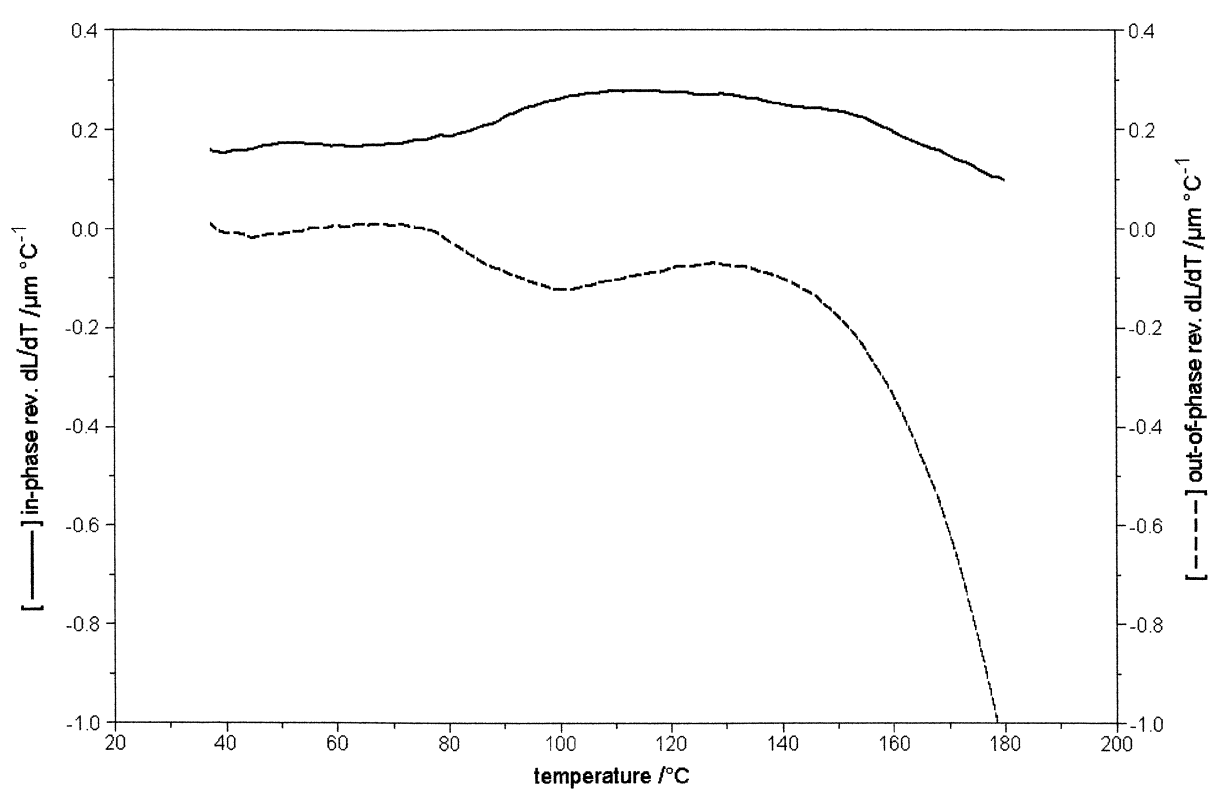

Fig. 3. In-phase and out of phase components of reversing $d L / d T$ from Fig. 2. 


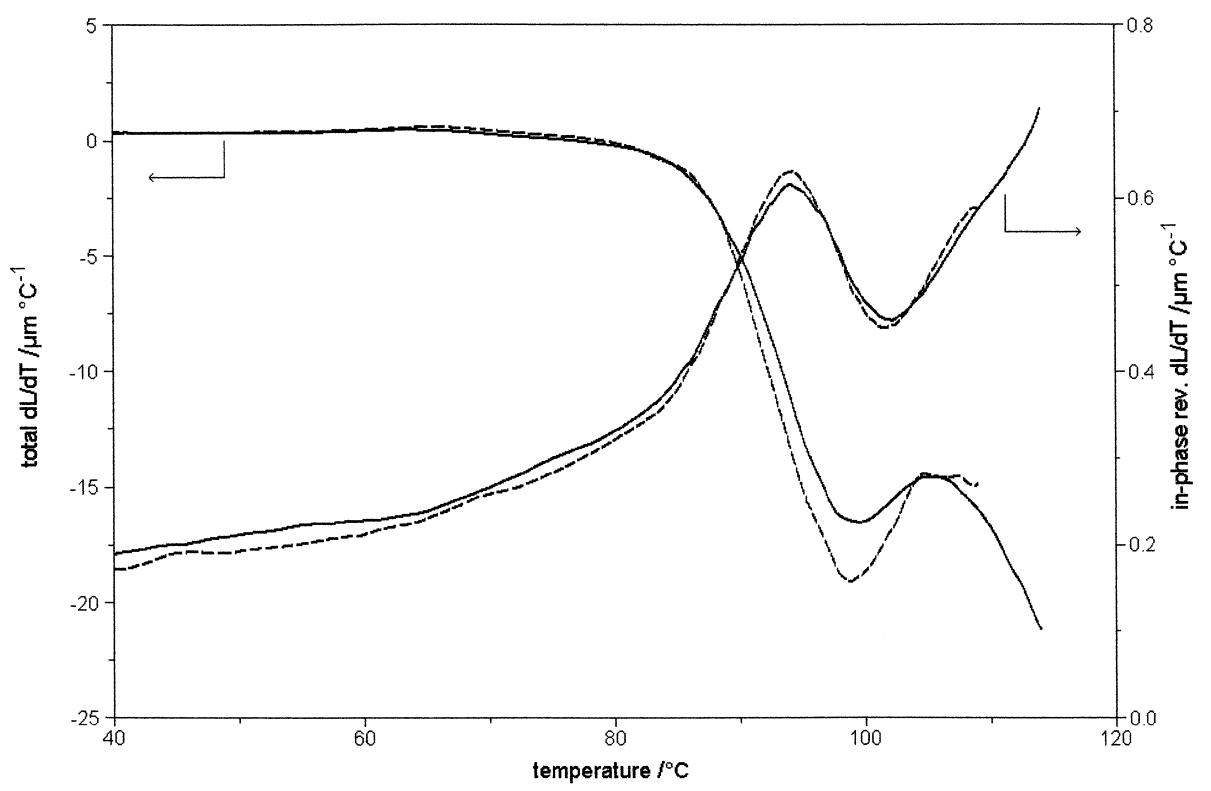

Fig. 4. MTTMA of poly(methyl methacrylate) rod in compression (solid line: $5 \mathrm{~g}$ load, dashed line: $10 \mathrm{~g}$ load).

above $T_{\mathrm{g}}$ due to relaxation effects [15]. At higher temperatures, there is an apparent reduction in the in-phase reversing $\mathrm{d} L / \mathrm{d} T$ due to non-linear response of the sample as it undergoes large-scale irreversible deformation during the time-scale of the temperature modulation. Such effects have been discussed in MTDSC [16] and may be overcome by using shorter modulation periods and lower modulation amplitudes. Separation of the reversing rate of length change into in-phase and out-of-phase components is also important for measurements in compression (where creep occurs) and allows the thermal expansion coefficients of materials to be evaluated independently of loading conditions (Fig. 4).

The rate of length change data may be integrated with respect to time (for the average $\mathrm{d} L / \mathrm{d} t$ ) or temperature (for the reversing $\mathrm{d} L / \mathrm{d} T$ ) to allow the total length change and length change due to thermal expansion to be determined. The difference between such curves represents the changes in dimensions due to irreversible shrinkage arise from loss of orientation or creep under load. This approach has been used to study orientation in PET films [10] and acrylic fibres [17].

When the sample is subjected to a dynamic load this technique becomes modulated-temperature dynamic mechanical analysis (MTDMA). Fig. 5 shows the results of an experiment performed on an epoxy resin (Ciba Aradite ${ }^{\circledR}$ ) rod in compression. In this case, the sample was heated in a scanning modulated-temperature fashion to $100^{\circ} \mathrm{C}$ and then the conditions were changed to quasi-isothermal operation. The data was first analysed to determine the amplitude and phase difference of the length change due to the applied loading cycle. This yields the complex compliance (from the ratio of the length change amplitude divided by the load amplitude) and damping factor (tan delta). Fig. 5 shows the average temperature profile, length and mechanical properties. The partially cured resin devitrifies and softens up to $50^{\circ} \mathrm{C}$, undergoes further cure up to $80^{\circ} \mathrm{C}$ and then softens once more.

The length change response was deconvoluted to determine reversing $\mathrm{d} L / \mathrm{d} T$ response and phase lag (Fig. 6). The changes in thermal expansivity closely follow the compliance. Furthermore, it is possible to measure the thermal expansion under quasi-isothermal conditions analogous to MTDSC [18]. Schick [19] has used MTDMA in both temperature-scanning and quasi-isothermal modes to study the reversible melting of polymers. MTDMA would appear to be complimentary to MTDSC in the study of curing systems [20]. 


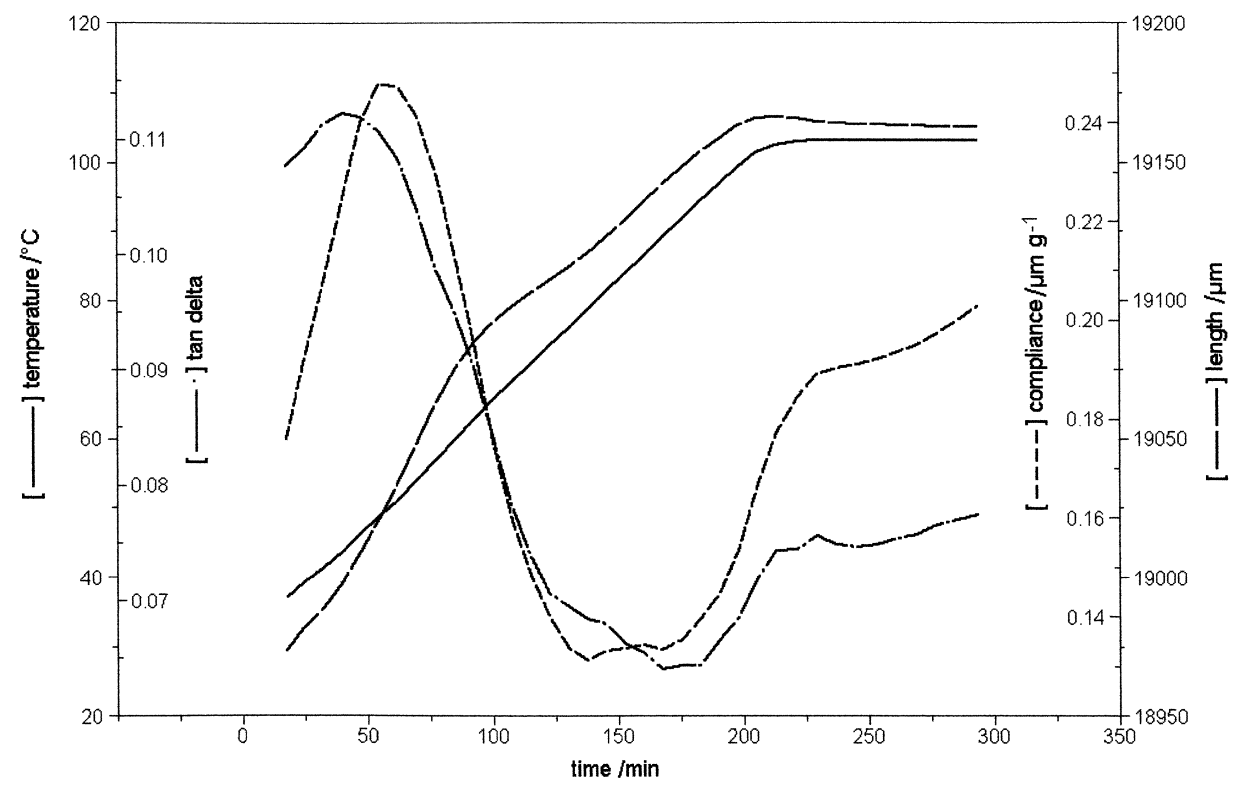

Fig. 5. MTDMA experiment on epoxy resin rod (temperature, length, compliance and damping factor).

Where the temperature program exposes the sample to cooling conditions in addition to heating, the data may be broken down into heat/cool/re-heat segments and analysed in the same way (Reading [12] has termed this 'parsing'). An example of this is shown in Fig. 7 for an acrylic fibre (Courtaulds Courtelle ${ }^{\mathbb{R}}$ ). The data are equivalent below the $T_{\mathrm{g}}\left(90^{\circ} \mathrm{C}\right)$. Above this temperature the effects of thermal history become

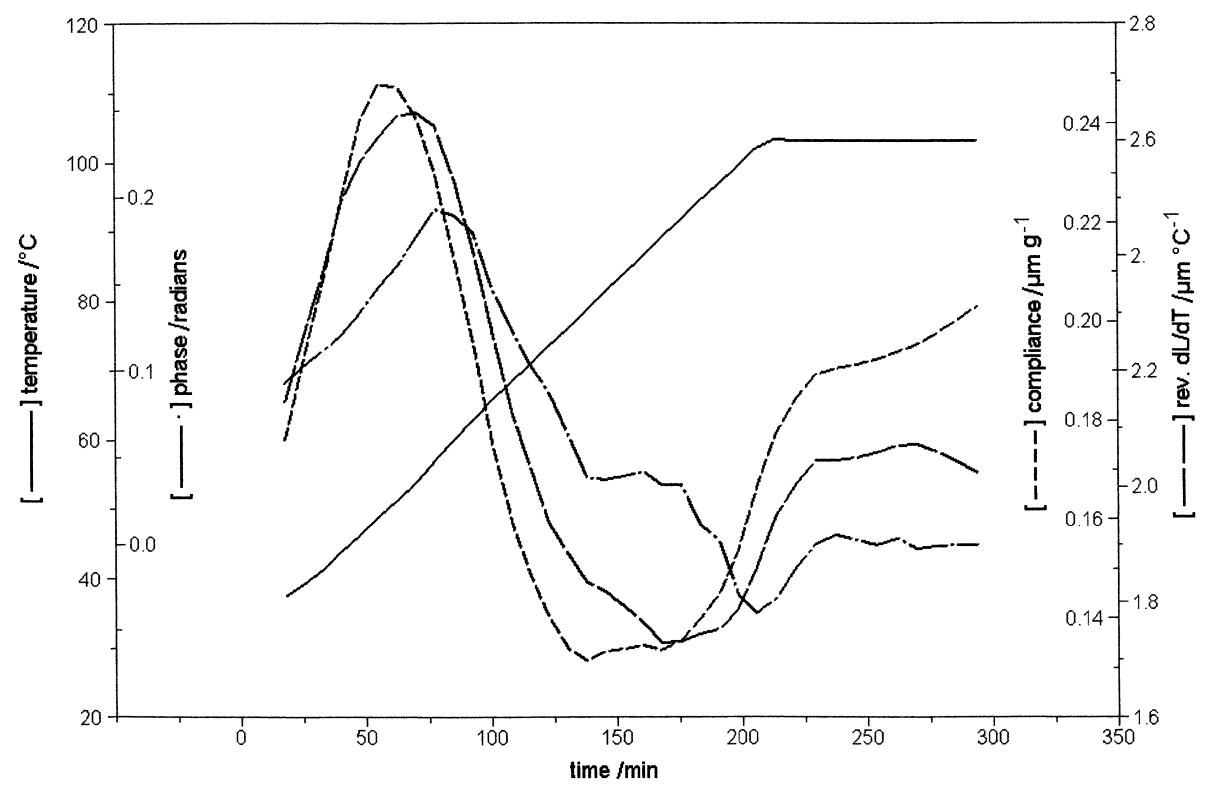

Fig. 6. MTDMA experiment on epoxy resin rod (temperature, reversing $\mathrm{d} L / \mathrm{d} T$, compliance and phase lag). 


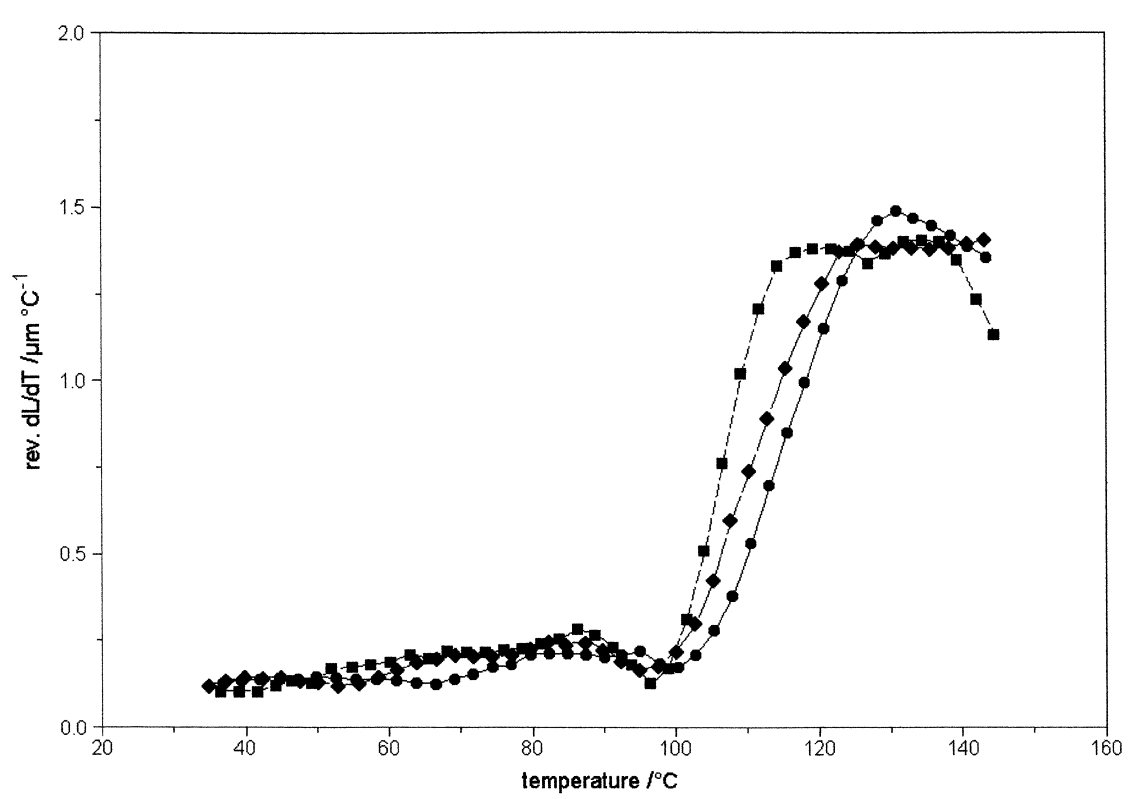

Fig. 7. Reversing $d L / d T$ separated into heat ), cool ( and re-heat ( components for an acrylic fibre.

apparent. For dynamic load experiments, it might be possible to isolate data depending on the direction of load change (e.g., creep versus stress relaxation) in addition to any temperature modulation.

\section{Conclusions}

These examples show the potential of modulatedtemperature programming for the study of dimensional changes with thermomechanical analysis. The effects of orientation and creep can be studied and the measurements can be made less sensitive to choice of initial load. The application of this approach to dynamic mechanical analysis presents interesting possibilities for studying cross-linking reactions and melting. Quasi-isothermal measurements of thermal expansivity can be made and 'parsing' may be utilised to investigate the effects of thermal history on the sample.

\section{Acknowledgements}

This work was carried out at the Thermal Methods and Conservation Science Laboratory, Department of
Chemistry, Birkbeck College, University of London. The loan of equipment by Shimadzu, UK, is acknowledged. The author would like to thank M. Odlyha and G.M. Foster (Birkbeck College) for assistance and M. Reading (Loughborough University) for helpful discussions.

\section{References}

[1] P.S. Gill, S.R. Sauerbrunn, M. Reading, J. Therm. Anal. 40 (1993) 931.

[2] M. Reading, D. Elliott, V.L. Hill, J. Therm. Anal. 40 (1993) 949.

[3] M. Reading, Trends Polym. Sci. 1 (1993) 248.

[4] M. Reading, A. Luget, R. Wilson, Thermochim. Acta 238 (1994) 295.

[5] J.E.K. Schawe, Thermochim. Acta 271 (1996) 127.

[6] R. Reisen, G. Widmann, R. Truttmann, Thermochim. Acta 272 (1996) 27.

[7] R. Blaine, Am. Lab. 30 (1998) 21.

[8] M. Jaffe, in: E.A. Turi, (Ed.), Thermal Characterization of Polymeric Materials, Academic Press, Orlando, 1981, p. 731.

[9] M. Trznadel, M. Kryszewski, J. Macromol. Sci. C32 (3\&4) (1992) 259.

[10] D.M. Price, J. Thermal Anal. 51 (1998) 231.

[11] D.M. Price, Thermochim. Acta 315 (1998) 11.

[12] M. Reading, US Patent 5,474,385, 12 December 1995.

[13] J.E.K. Schawe, Thermochim. Acta 260 (1995) 1. 
[14] M. Reading, Thermochim. Acta 292 (1997) 179.

[15] B. Howarth, Z.W. Dong, P. Davidson, Polym. Int. 32 (1993) 325.

[16] T. Ozawa, K. Kanari, Thermochim. Acta 253 (1995) 183.

[17] D.M. Price, G.M. Foster, J. Thermal Anal. Cal. 56 (1999) 649.
[18] A. Boller, Y. Jin, B. Wunderlich, J. Thermal Anal 42 (1994) 307.

[19] A. Wurm, M. Merzlyakov, C. Schick, Colloid Polym. Sci. 276 (1998) 289.

[20] G. Van Assche, A. Van Hemelrijck, H. Rahier, B. Van Mele, Thermochim. Acta 268 (1995) 121. 